\title{
Cooperation in neural systems: Bridging complexity and periodicity
}

\author{
Marzieh Zare and Paolo Grigolini \\ Center for Nonlinear Science, University of North Texas, P.O. Box 311427, Denton, Texas 76203-1427, USA
}

(Received 31 July 2012; revised manuscript received 22 October 2012; published 29 November 2012)

\begin{abstract}
Inverse power law distributions are generally interpreted as a manifestation of complexity, and waiting time distributions with power index $\mu<2$ reflect the occurrence of ergodicity-breaking renewal events. In this paper we show how to combine these properties with the apparently foreign clocklike nature of biological processes. We use a two-dimensional regular network of leaky integrate-and-fire neurons, each of which is linked to its four nearest neighbors, to show that both complexity and periodicity are generated by locality breakdown: Links of increasing strength have the effect of turning local interactions into long-range interactions, thereby generating time complexity followed by time periodicity. Increasing the density of neuron firings reduces the influence of periodicity, thus creating a cooperation-induced renewal condition that is distinctly non-Poissonian.
\end{abstract}

DOI: 10.1103/PhysRevE.86.051918

PACS number(s): 87.16.dj, 05.45.Tp, 05.65.+b, 87.19.1j

\section{INTRODUCTION}

The emergence of scale-free distributions from phase transitions affords a promising way to define complexity, which is the subject of a rapidly developing field of investigation, still in its infancy [1]. According to a widely shared theoretical interdisciplinary perspective, ranging from neurophysiology to sociology, from geophysics to economics, heavy tails and inverse power law distributions [2,3] are thought to be the signature of complexity. Of special interest are the scale-free distributions in time, for instance, the waiting time distributions of the "light on" and "light off" states of intermittent fluorescence in quantum dots [4-7], which has been proved [8,9] to be a renewal non-Poisson process. These scale-free waiting time distribution densities have the time-asymptotic form

$$
\psi(t) \propto \frac{1}{t^{\mu}}
$$

Hence the corresponding cumulative distributions, or survival probabilities, yield the very slow decay

$$
\Psi(t) \equiv \int_{0}^{\infty} \psi(t) d t \propto \frac{1}{t^{\mu-1}} .
$$

Experimental observation shows that $\mu<2$, thereby making the mean waiting time distribution diverge, with a consequent violation of ergodicity. The connection between temporal complexity and ergodicity breaking has stimulated the extension of fundamental theoretical tools such as the invariant density [10], the Kolmogorov-Sinai entropy [11], and the Khinchin theorem [12]. Other interesting aspects of ergodicity breaking in condensed matter are illustrated in Refs. [13-15].

Neural avalanches, with scale-free distribution in both size and time duration, are another significant manifestation of natural complexity emerging from criticality [16-21]. The hypothesis of criticality affords an attractive way of explaining the intelligent global behavior of cooperative systems. The conversion of local into long-range interactions generated by criticality, termed cooperation-induced long-range interaction, was recently proved to yield an efficient way to transport information [22] and may be used to explain the zero time lag synchronization among remote cerebral cortical areas [23].
Furthermore, criticality-induced fluctuations are non-Poisson renewal processes [24], namely, close relatives of the complex dynamical processes of Refs. [10-15], in accordance with the earlier work of Refs. [25,26].

As attractive as this interdisciplinary perspective of complexity may be, it is not clear to what extent it is compatible with the intrinsic periodicity of biological processes [27]. This is a subject attracting increasing attention, as proved by the recent remarkable work of Ref. [28], which does, however unexplored the periodicity-criticality issue.

The main purpose of this paper is to show, with the help of a model of cooperating neurons, that periodicity may emerge naturally from the criticality-induced long-range correlations. As in Refs. [22,23], criticality breaks the statistical independence of two neurons, regardless of their Euclidean distance, For larger values of the cooperation parameter, this eventually yields perfect synchronization and periodicity.

The outline of this paper is as follows. Section II describes the neural model of this paper. It is a rigorously local version of the model of the earlier work of Ref. [29]. Section III illustrates the form of temporal complexity generated by the cooperative interaction between the units of this model. Section IV focuses on a technique of statistical analysis that is used to establish the influence of periodicity on temporal complexity. It shows that in the low-density case, when the slow decay of the survival probability makes the system perceive periodicity, the renewal condition is broken and the neural quakes become predictable. Section $V$ discusses the size distribution of neural quakes and shows that the adoption of the distribution density rather than the cumulative distribution makes the influence of periodicity on the avalanche statistics visible, although affording less precise information on the power law indices. We make concluding remarks in Sec. VI.

\section{COOPERATIVE NEURAL NETWORK}

We use the popular Leaky Integrate-and-Fire Model (LIFM) [30], in the presence of a noise of intensity $\sigma^{2}$ [31,32]. The LIFM is described by

$$
\frac{d}{d t} x=-\gamma x(t)+S+\sigma \xi(t),
$$


where $x$ is the cell membrane potential, $S$ is a positive drift to the firing threshold $\Theta=1,1 / \gamma(\gamma<S)$ is a constant governing the decay of the voltage back to the resting level $x=0$, and $\xi(t)$ is a random fluctuation with $\left\langle\xi^{2}(t)\right\rangle=1$. All these variables are dimensionless. When $x(t)$ reaches the threshold value, the neuron fires and returns to its resting potential. We study a set of $N$ LIFM neurons and for the numerical solution of Eq. (3), we adopt the integration time step $\Delta t=1$, with $\gamma \ll 1$ and $S \ll 1$. With Mirollo and Strogatz [33] we assume that the firing of one neuron increases the position of all the neurons linked to it from the value $x(t)$ established by Eq. (3) to $x(t)+K$, with $0<K \ll 1$. We refer to this model herein as the cooperative LIFM.

When $K=0$, the vanishing noise condition yields for the time distance between two consecutive firings of the same neuron the following expression [31]:

$$
T_{P}=\frac{1}{\gamma} \log \left(\frac{1}{1-\frac{\gamma}{S}}\right) .
$$

If $K>0$, after a few time steps all the neurons fire at the same time [33], thereby generating a sequence of quakes of intensity $N$ with the time period $T_{P}$ of Eq. (4). This is the periodicity and synchronization limiting condition to be contrasted to the opposite limiting case of no correlation between the neurons. The demonstration of Ref. [33] is based on the All-To-All (ATA) coupling condition and consequently misses the cooperation-induced locality breaking.

In this article instead we represent the neuronal network as a two-dimensional regular lattice with periodic boundary conditions, where each neuron is linked only to its four nearest neighbors, and thus has only four links rather than the $N-1$ of Ref. [33]. The adoption of periodic boundary conditions is dictated not only by the purpose of simulating a system of more extended size, but also to ensure the total equivalence of the cooperating units, so as to avoid the doubt that the onset of firing bursts may be triggered by units with a favorable topology. It is remarkable that with large enough values of $K$, the cooperative system of this article realizes the same periodic behavior of Ref. [33], where the neuron equivalence is automatically realized by the ATA condition. Numerical calculations not reported here show that the adoption of periodic boundary condition is not crucial for the results of this paper.

In other words, we prove that cooperation generates longrange interactions, a property proved to be essential for the function of a swarm [22], and with a conveniently large $K$ even the perfect synchronization of Ref. [33]. The equivalence of the model of this article with the ATA condition of Ref. [33], for much larger values of $K$, indicates that when the periodicity condition is realized, the cooperative system breaks locality. We show that to realize periodicity the system has to cross complexity that emerges at intermediate values of $K$.

\section{TEMPORAL COMPLEXITY}

As pointed out above, it is necessary to activate the noise $\xi(t)[34,35]$ to generate temporal complexity. Temporal complexity is compatible with the quasiperiodicity of the single-neuron dynamics. If noise intensity is conveniently small, the time spent by one neuron to move from rest to the threshold potential remains close to $T_{P}$ of Eq. (4). Although this condition is ensured by setting $\sigma<\sqrt{\gamma}$, making the mean time distance between two consecutive firings of the same neuron very close to $T_{P}$, in the absence of cooperation periodicity is totally lost. This is a consequence of the noise action. For very low values of $K$, the dynamics of the whole system are determined by the uncorrelated motion of many units, with the time scale $T_{P}$. Due to the lack of correlation, the time distance between two consecutive firings of a set of $N$ neurons is given by $G=\frac{N}{\langle\tau\rangle}$ with $\langle\tau\rangle \approx T_{P}$. As a consequence, the survival probability $\Psi(t)$, namely, the probability that no firing occurs up to the time $t$ from an earlier firing, is given by

$$
\Psi(t)=e^{-G t},
$$

which is the typical form of Poisson dynamics. According to the conviction of Ref. [1] that cooperation generates scale invariance, one would expect as an effect of increasing $K$ a transition from the exponential form of Eq. (5) to

$$
\Psi_{C}(t)=\left(\frac{T_{C}}{T_{C}+t}\right)^{\alpha},
$$

where $\alpha \equiv \mu-1$, with $T_{C}$ so small as to make Eq. (6) virtually equivalent to the inverse power law of Eq. (1) over the available time scale. As shown by Fig. 1, we find instead that for small values of $N$, the survival probability is identical to the MittagLeffler (ML) function [36-38]

$$
\Psi(t)=E_{\alpha}\left[-\left(\lambda_{\alpha} t\right)^{\alpha}\right]
$$

if we neglect the periodicity-induced long-time truncation. As explained by the authors of Ref. [36], the ML function plays an important role in the field of complexity because it settles the controversy between the advocates of stretched exponential functions and the advocates of inverse power law as important signatures of complexity. In fact, in the time region $t<1 / \lambda_{\alpha}$ the ML survival probability is described by the stretched exponential function

$$
\Psi(t) \propto \exp \left[-\left(\lambda_{\alpha} t\right)^{\alpha}\right]
$$

and in the large time region $t>1 / \lambda_{\alpha}$ by

$$
\Psi(t) \propto \frac{1}{t^{\alpha}} .
$$

The cooperation-induced emergence of ML function matches the expectation that cooperation generates scale invariance. In fact, the index $\alpha$ of the stretched exponential function of Eq. (8) is identical to the power index $\alpha$ of the inverse power law of Eq. (9). On top of that we show that the ML function of Eq. (7) is the visible manifestation of a hidden survival probability with the inverse power law form of Eq. (6), which is thought to be a signature of complexity [1].

To prove this important fact, we notice that as an effect of cooperation the neurons tend to fire at the same time [29,39], thereby making the distance between two consecutive firings become larger than $1 / G$ and the intensity of each firing larger than the intensity of a single firing. As a consequence of the fact that the neural network has a finite number $N$ of units, some of these multiple firings cannot be realized, or, let us say, they are not visible. If we increase $N$ while leaving $T_{P}$ 

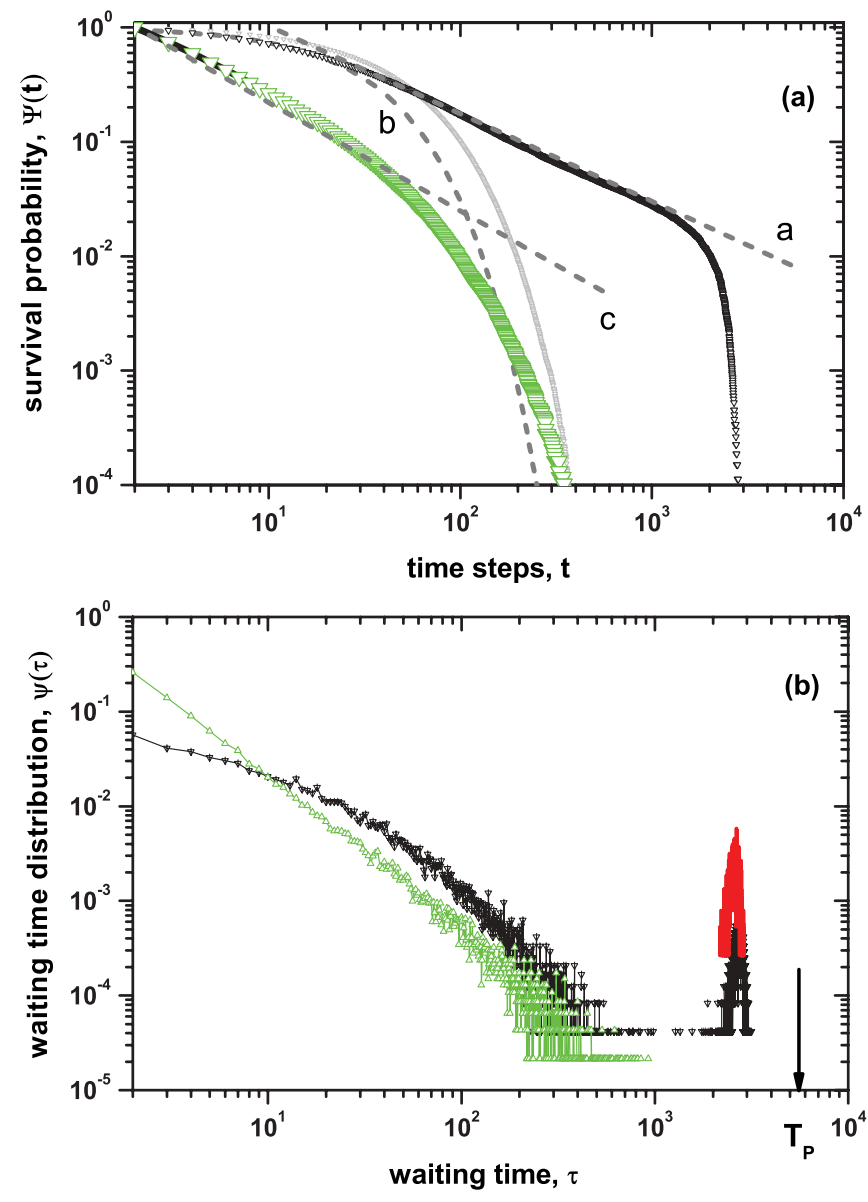

FIG. 1. (Color online) (a) Survival probability $\Psi(t)$. All the curves refer to $\sigma=0.0006, S=0.001, \gamma=0.001005$. Thin gray curve: $K=0, N=100$; black curve: $K=0.09, N=100$; thick green curve: $K=0.09, N=2500$. The dashed line (a) at long-time regime and the dotted line (b) short-time regime have been obtained using the Laplace transform fitting technique of Refs. [29,39]. The former is the stretched exponential function $\exp [-(\lambda t)]^{\alpha}$ with $\lambda=0.027$ and $\alpha=0.75$ corresponding to Eq. (6). The latter denotes $1 / t^{\alpha}$ with the same $\alpha$ as the short-time stretched exponential, Eq. (7). The dash-dotted line (c) has been obtained by shifting down the line (a). (b) Waiting time distribution $\psi(t)$. The green and black curve lines have the same parameters as the corresponding curves of panel a. Red curve: $K=0.4, N=100$. The vertical arrow denotes $t=T_{P}$.

constant, thereby increasing the density of neuron firings, all the cooperation-induced multiple firings are realized and are visible, and they are expected to generate a complex survival probability $\Psi_{C}(t)$. Note that this form of temporal complexity yields the ergodicity breaking of Refs. [10-15] when $\alpha<1$.

However, for $N$ small, many firings are not realized. Let us denote by $P_{S}$ the probability that a multiple firing occurs. Decreasing $N$ makes $P_{S}$ decrease so that $\Psi_{C}(t)$, as shown in Ref. [39], is replaced by the much slower survival probability of Eq. (7) with

$$
\lambda_{\alpha}=\left[\frac{P_{S}}{\Gamma(1-\alpha)}\right]^{1 / \alpha} \frac{1}{T_{C}},
$$

which shows that with $P_{S} \rightarrow 0, \Psi(t)$ does not decay, signaling that no event occurs. In the ideal case of perfect synchronization studied by Mirollo and Strogatz [33] the system produces a sequence of firings at times $n T_{P}$, with $n=1,2, \ldots$ with intensity equal to $N$. When $K=0, N$ firings of minimal intensity, with only one neuron firing, are homogeneously distributed in the time region between $(n-1) T_{P}$ and $n T_{P}$. For intermediate values of $K$ the density of firings in these time intervals decreases, and if one of these time intervals is empty, with firings of intensity $N$ at both extremes, it will contribute a peak of the waiting time distribution $\psi(t)$ at $t \approx T_{P}$. The intensity of this peak is proportional to the number of empty time intervals of length $T_{P}$. Actually, as a consequence of working with $\sigma>0$, the time length of the largest empty time intervals will be smaller than $T_{P}$, but these peaks will still perceive the system's periodicity. If the survival probability $\Psi(t)$ virtually vanishes at $t \ll T_{P}$, as in the high-density case, generating the survival probability of Eq. (6) with $T_{C} \ll T_{P}$, the influence of periodicity is not perceived, and the intensity of the truncation peaks should be minimal.

Figure 1 fully supports these theoretical arguments shows that lower density yields a slow and heavy tail ML survival probability. The fast drop in the long-time region close to $T_{P}$ is due to the high sensitivity to periodicity (panel a). It is important to notice that the ML function revealed by the numerical analysis of this article exactly matches the ATA results [29]. This is strong evidence that cooperation generates long-range interactions, thereby making the local-interaction network identical to the ATA network, if a larger $K$ is adopted: Cooperation-induced criticality breaks the local nature of the model [22]. The low-density $(N=100)$ corresponding waiting time distribution [Fig. 1(b)] shows a large peak at $t \approx T_{P}$, while the high-density one $(N=2500)$ shows no sign of truncation peak, in agreement with the earlier theoretical arguments. For illustrative purposes we have also plotted the perfect synchronization case of $K=0.4$. It is important to stress that the power law regime of the survival probability of the high-density case is too limited to allow us to use a reliable fitting procedure to find a power index. Line (b) is a guideline obtained by shifting down the fitting line (a) with the power index of $\alpha=0.75$. The reasons of the drop of this power law are not clear. They may be due to random fluctuations influencing the extended transition time regime from $10^{2}$ to $10^{3}$. The transition time is not affected by periodicity as clearly proved by the aging experiment (see Fig. 2), ensuring that the process is still renewal.

\section{COOPERATION-INDUCED RENEWAL BREAKING}

Let us now address another key issue of this article, the periodicity-induced renewal breaking. The model of Eq. (3) in the absence of stimuli is renewal, since after firing a neuron does not have any memory of the earlier paths. However, under the presence of a harmonic stimulus it may lose its renewal properties [31,32]. The model of this paper, the cooperative LIFM, can be turned into a nonrenewal process without external stimuli, due to the new phenomenon of cooperationinduced renewal breaking. To detect the renewal breaking and to measure its intensity, we adopt the aging experiment that was designed some years ago to establish the renewal nature of the 


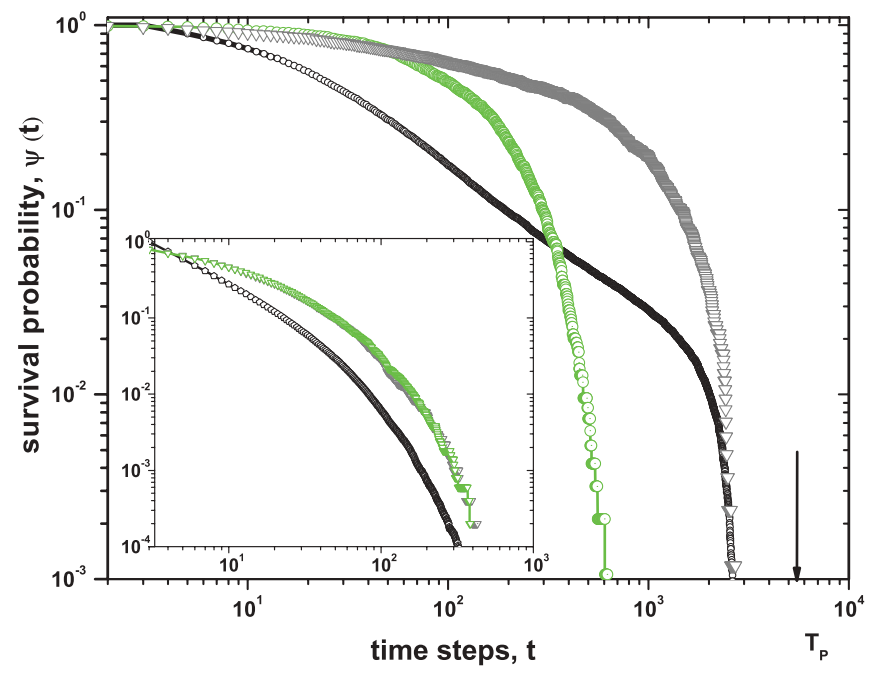

FIG. 2. (Color online) Aging experiment for the survival probability in the low-density case, $N=100$. The black curve has the same parameters as those of the black curves of Fig. 1 with $t_{a}=0$. The gray and green curves are the survival probabilities of age $t_{a}=3000$ with and without shuffling, respectively. Inset: Aging experiment for the survival probability in the high-density case, $N=2500$; black curve has the same parameters as those of the green curves of Fig. 1 with $t_{a}=0$.The virtually coinciding gray and green curves are the survival probabilities of age $t_{a}=300$ with and without shuffling, respectively.

blinking quantum dots phenomenon [9]. To explain how this method of statistical analysis works and to highlight its broad range of applications, let us adopt the perspective advocated by Osorio et al. [40] establishing a surprising analogy between geophysical and neurophysiological quakes. Let us imagine that each firing is a quake, and let us address the issue of assessing if these quakes are predictable or not. We record the time $t_{i}$ of occurrence of each quake, and we generate the time series $\left\{\tau_{i}\right\} \equiv \tau_{1}, \tau_{2}, \ldots, \tau_{i}, \ldots$, where $\tau_{i} \equiv t_{i+1}-t_{i}$. We generate also an auxiliary sequence $\left\{\tau_{i}\right\} \equiv \tau_{1}^{\prime}, \tau_{2}^{\prime}, \ldots, \tau_{i}^{\prime}$ by shuffling the sequence $\left\{\tau_{i}\right\}$. The time of occurrence of the quakes of the auxiliary sequence $t_{1}^{\prime} \equiv \tau_{1}^{\prime}, t_{2}^{\prime} \equiv \tau_{1}^{\prime}+$ $\tau_{2}^{\prime}, \ldots, t_{i}^{\prime} \equiv \tau_{1}^{\prime}+\cdots \tau_{i-1}^{\prime}+\tau_{i}^{\prime}$ are totally unpredictable, as a consequence of the random shuffling. Let us now imagine that the time region between two consecutive quakes are assigned either the value $\zeta=1$ or -1 according to a coin tossing prescription. We thus obtain the dichotomous fluctuation $\zeta(t)$ that in the case of the shuffled sequence is a renewal process. In the ordinary case where the process is renewal and the waiting time distribution density $\psi(\tau)$ is exponential, the correlation function $\left\langle\zeta\left(t_{2}\right) \zeta\left(t_{1}\right)\right\rangle$ is a stationary and exponential correlation function. In the case of the model of this paper, this is the weak-coupling condition, where the waiting time distribution density corresponding to the survival probability of Eq. (5) is $\psi(\tau)=G \exp [-G \tau)]$. In the non-Poisson case the correlation function $\left\langle\zeta\left(t_{2}\right) \zeta\left(t_{1}\right)\right\rangle$ becomes nonstationary [4,41], and for this reason it is convenient to write it as $\left\langle\zeta\left(t_{2}\right) \zeta\left(t_{1}\right)\right\rangle=\Phi_{\zeta}\left(\tau, t_{a}\right)$, with $\tau \equiv t_{2}-t_{1}$ and $t_{a}=t_{1}$. It is expected that the decay of this correlation function becomes slower and slower with its age, namely upon increasing $t_{a}$. In principle the correlation function should be evaluated by the ordinary Gibbs ensemble average.
Following Ref. [9] we evaluate this nonstationary correlation function by means of a moving time window of size $t_{a}$, with the left end located on the time of occurrence of a quake. We evaluate the time distance between the right end of the window and the first quake after it. The corresponding waiting time distribution is normalized, and its survival probability coincides with the nonstationary correlation function $\Phi_{\zeta}\left(\tau, t_{a}\right)$ [42]. With the use of this procedure, we obtain the central result of Fig. 2 illustrating the case where $K>0$ and $N$ is so small as to reduce the number of visible multiple firings and yield the slow ML survival probability of Fig. 1(a), quickly dropping when $t$ has the same order of magnitude as $T_{P}$. We see that the shuffled and the original sequence undergo the same aging effect in the time region $t \ll T_{P}$, whereas in the remaining wide time region the aging of the shuffled sequence departs significantly from that of the original sequence. This is a clear effect of renewal-breaking periodicity. The quakes of the former time region are renewal and unpredictable, whereas in the latter time region they may be predictable. Of course, when $K$ is very large as to fit the condition of perfect synchronization of Mirollo and Strogatz [33] the whole process becomes perfectly predictable. The inset of Fig. 2 supports the claim that the high-density case is insensitive to periodicity. In
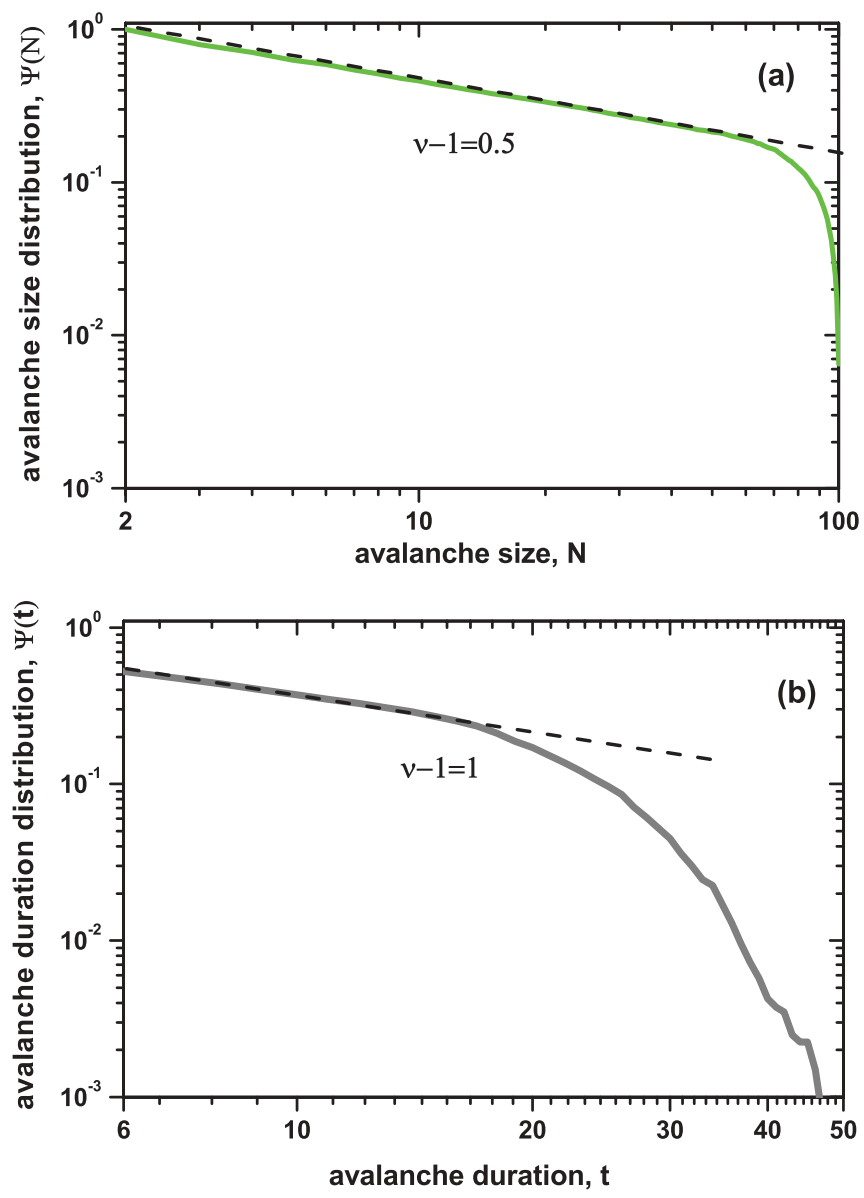

FIG. 3. (Color online) (a) Green line shows the avalanche size distribution, $N=100$ and $K=0.12$. (b) Gray line shows avalanche time duration distribution for the same values of $\mathrm{N}$ and $\mathrm{K}$. The dashed lines in each panel correspond to the power law indexes $\alpha=1.5$ (a) and $\alpha=2$ (b), respectively. 


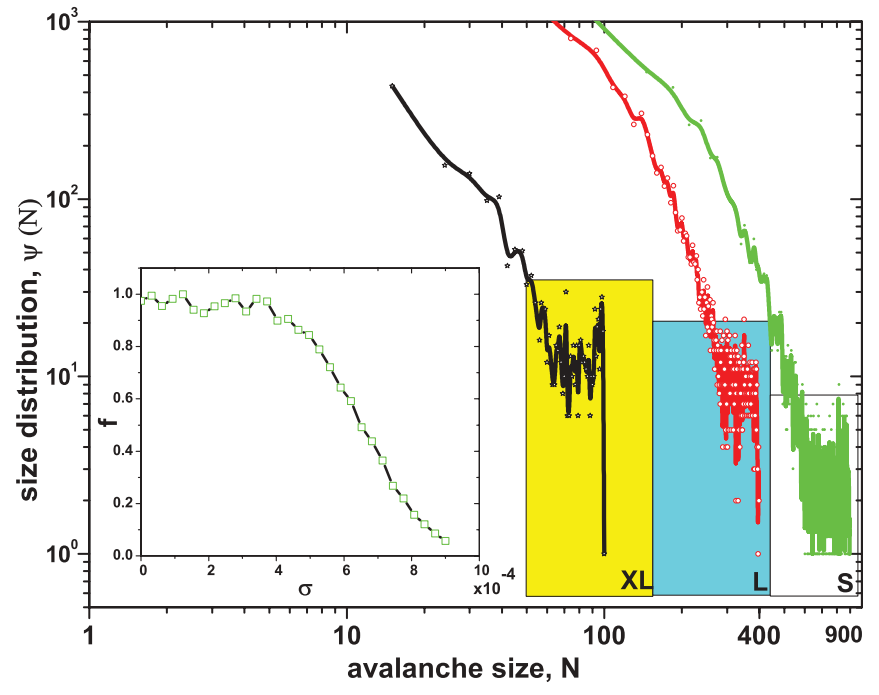

FIG. 4. (Color online) Avalanche size distribution for $N=100$ (black curve), $N=400$ (red curve), and $N=900$ (green curve). In all three cases $\sigma=0.0006$ and $K=0.12$. Data are log-binned. Inset: Fraction of the number of the avalanches of intensity $N$ to the total number of avalanches, as a function of $\sigma$.

fact, there is aging, and so a strong departure from the Poisson condition, while the virtually perfect coincidence between the results of shuffled and nonshuffled procedures proves that the process is renewal. In this ideal case of ordinary complexity, the avalanches are totally unpredictable.

\section{NEURAL AVALANCHES}

We note that the results of Fig. 1 do not afford any direct information on the quake intensity. To discuss this problem, we study the distribution of avalanche sizes for different values of $N$. Avalanches are a well-known property of neural networks and the model studied in this article generates avalanch distribution with power indices matching the experimental observation [16], 1.5 for the avalanche size. Figure 3(a) shows that the model of this paper fits this property, as already done in Ref. [39]. Figure 3(b) shows that the power index of avalanche time duration distribution is 2 , in accordance with the earlier result of Ref. [29]. It is convenient to reiterate that the result of Ref. [29] rests on the ATA condition, and recovering this property in the two-dimensional case adds further support to one of the important results of this paper, about the cooperation-induced locality breaking.

Here we focus on the size distribution. Figure 4 shows that the distribution density of avalanche sizes for a suitably large value of $K$ is characterized by a bump, and this bump becomes larger and larger with decreasing $N$, a property shared also by the model used by de Arcangelis [43]. The truncation peak corresponds to the ideal synchronization of Mirollo and Strogatz [33], namely, to an avalanche of maximal intensity $N$. Setting $\sigma=0$ prevents small avalanches to unfold, a limiting condition of periodicity with no temporal complexity, which according to the inset of Fig. 4 extends to a small range of $\sigma$ values. Gigantic avalanches of size $N$ may coexist with temporal complexity and with the experimental power law distribution of index $\mu=1.5$, and the fraction of avalanches of maximal intensity emerging from a power law background becomes larger with decreasing the neuron density, due to the close connection between sensitivity to avalanches and the heavy tails of $\Psi(t)$.

\section{CONCLUDING REMARKS}

The neural model of this paper sheds light into the coexistence of periodicity and complexity. In spite of the fact that each neuron interacts only with four nearest neighbors, suitably large values of $K$ make this model recover the perfect synchronization of Ref. [33]. However, an extended regime exists between the weak-coupling regime, where the units of the system are independent of each other, and the perfect synchronization regime. In this intermediate regime, periodicity and complexity coexist.

This may contribute to the foundation of a theory for the occurrence of Dragon Kings proposed by Sornette, a new phenomenon that is currently the object of a vigorous debate [43-45]. The Dragon Kings are anomalous events emerging from a power law background. This paper establishes a connection between the existence of Dragon Kings and the apparently conflicting coexistence of renewal and nonrenewal properties in the same complex model. Interpreting the Dragon Kings as outliers [44] generates the impression that they are foreign to the cooperation-induced locality breaking from which temporal complexity emerges. We make the conjecture that the peaks emerging at the maximum value of avalanche size distribution $N$ coinciding with the total number of neurons are the Dragon Kings of Refs. [43-45]. If this interpretation is correct, the Dragon Kings would be a manifestation of the same cooperation-induced long-range correlation as that proved to be essential for the function of complex systems [22]. The authors of the earlier work of Ref. [29] studied the ATA version of this model and interpreted its criticality as a form of extended criticality [46]. Further investigation is necessary to refine this conclusion. Here we limit ourselves to noticing that the wide complex regimes, where temporal complexity and periodicity coexist, extend from the Poisson to the perfect synchronization regime and that the latter regime is a manifestation of cooperation-induced long range correlation.

Last but least, we want to point out that the results obtained in this paper may be general. The adoption of a regular two-dimensional network has been decided as the simplest way to assign to each neuron the same number of links. On the basis of the results of the recent work of Ref. [47] we make the plausible conjecture that the adoption of different network topologies generates temporal complexity, locality breakdown, and perfect synchronization upon changing the values of $K$. There may be scale-free networks where all the results of this paper are recovered at lower values of $K$. In fact, the work of Ref. [47] shows that the cooperative system generates dynamical links with a scale-free structure that has the important effect of facilitating the transition from the Poisson condition to the criticality-induced long-range correlation regime. However, while the cooperative model used in Ref. [47] is Ising-like, and is renewal for both low and high values of the cooperation parameter [24], by contrast, 
the model of this paper shows a transition from the renewal condition (at the emergence of criticality) to a predictable nonrenewal coherent regime as the cooperation parameter $\mathrm{K}$ increases.

\section{ACKNOWLEDGMENT}

The authors gratefully acknowledge financial support from Welch and ARO through Grant N. B-1577 and W911NF-111-0478, respectively.
[1] K. Christensen and N. R. Moloney, Complexity and Criticality (Imperial College Press, London, 2005).

[2] P. Bak, C. Tang, and K. Wiesenfeld, Phys. Rev. Lett. 59, 381 (1987).

[3] J. Laherrè and D. Sornette, Eur. Phys. J. B 2, 525 (1998).

[4] F. D. Stefani, J. P. Hoogenboom, and E. Barkai, Phys. Today 2, 34 (2009).

[5] T. D. Krauss and J. J. Peterson, Nat. Mater. 11, 14 (2012).

[6] C. Galland, Y. Ghosh, A. Steinbrück, M. Sykora, J. A. Hollingsworth, V. I. Klimov, and H. Htoon, Nature (London) 479, 203 (2011).

[7] E. A. Riley, C. M. Hess, P. J. Whitham, and P. J. Reid, J. Chem. Phys. 136, 184508 (2012).

[8] X. Brokmann, J.-P. Hermier, G. Messin, P. Desbiolles, J.-P. Bouchaud, and M. Dahan, Phys. Rev. Lett. 90, 120601 (2003).

[9] S. Bianco, P. Grigolini, and P. Paradisi, J. Chem. Phys. 123, 174704 (2005)

[10] N. Korabel and E. Barkai, Phys. Rev. Lett. 108, 060604 (2012).

[11] N. Korabel and E. Barkai, Phys. Rev. Lett. 102, 050601 (2009).

[12] S. Burov, R. Metzler, and E. Barkai, Proc. Natl. Acad. Sci. USA 107, 13228 (2010).

[13] G. Bel and E. Barkai, Phys. Rev. Lett. 94, 240602 (2005).

[14] A. Lubelski, I. M. Sokolov, and J. Klafter, Phys. Rev. Lett. 100, 250602 (2008)

[15] Y. He, S. Burov, R. Metzler, and E. Barkai, Phys. Rev. Lett. 101, 058101 (2008)

[16] J. M. Beggs and D. Plenz, J. Neurosci. 24, 5216 (2004).

[17] A. Levina, J. M. Hermann, and T. Geisel, Nature Phys. 3, 857 (2007).

[18] D. R. Chialvo, Nature Phys. 6, 744 (2010).

[19] L. de Arcangelis, C. Perrone-Capano, and H. J. Herrmann, Phys. Rev. Lett. 96, 028107 (2006).

[20] S.-J. Wang and C. Zhou, New J. Phys. 14, 023005 (2012).

[21] J. M. Beggs and N. Timme, Front. Physiol. 3, 163 (2012).

[22] F. Vanni, M. Lukovic, and P. Grigolini, Phys. Rev. Lett. 107, 078103 (2011).

[23] R. Vicente, L. L. Gollo, C. R. Mirasso, I. Fisher, and G. Pipa, Proc. Natl. Acad. Sci. USA 105, 17157 (2008).

[24] M. Turalska, B. J. West, and P. Grigolini, Phys. Rev. E 83, 061142 (2011).

[25] P. A. Frantsuzov, S. Volkan-Kacso, and B. Janko, Phys. Rev. Lett. 103, 207402 (2009).

[26] S. Bianco, E. Geneston, P. Grigolini, and M. Ignaccolo, Physica A 387, 1387 (2008).
[27] A. T. Winfree, The Geometry of Biological Time, 2nd ed. (Springer-Verlag, New York, 2001). First edition published (1980).

[28] H.-H. Jo, M. Karsai, J. Kertész, and K. Kaski, New J. Phys. 14, 013055 (2012); M. Karsai, K. Kaski, A.-L. Barabàsi, and J. Kertész, Sci. Rep. 2, 397 (2012).

[29] E. Lovecchio, P. Allegrini, E. Geneston, B. J. West, and P. Grigolini, Front. Fractal Physiol. 98, 1 (2012).

[30] W. Gerstner and W. M. Kistler, Spiking Neuron Models (Cambridge University Press, Cambridge, 2002).

[31] A. R. Bulsara, T. C. Elston, C. R. Doering, S. B. Lowen, and K. Lindenberg, Phys. Rev. E 53, 3958 (1996).

[32] P. Lánský, Phys. Rev. E 55, 2040 (1997).

[33] R. E. Mirollo and S. H. Strogatz, SIAM J. Appl. Math. 50, 1645 (1990).

[34] Note that in the model of Ref. [35] randomness is introduced by assigning to the neurons different values of the parameter $S$. In Ref. [35] all the links are inhibitory, rather than excitatory as in the case of this paper, and the ATA coupling is adopted.

[35] S. Luccioli and A. Politi, Phys. Rev. Lett. 105, 158104 (2010).

[36] R. Metzler and J. Klafter, J. Non-Cryst. Solids 305, 81 (2002).

[37] F. Mainardi, J. Comp. Appl. Math. 118, 283 (2000).

[38] Note that the ML function is intimately related to the fractional calculus, widely used in different disciplines, ranging from physics to biology and to economics, and consequently is thought to be a important theoretical tool for the field of complexity.

[39] P. Grigolini, M. Zare, A. Svenkeson, B. J. West, Criticality in Neural Systems, edited by D. Plenz and E. Niebur, John Wiley, New York (in press).

[40] I. Osorio, M. G. Frei, D. Sornette, J. Milton, and Y. C. Lai, Phys. Rev. E 82, 021919 (2010).

[41] C. Godrèche and J. M. Luck, J. Stat. Phys. 104, 489 (2001).

[42] G. Aquino, M. Bologna, P. Grigolini, and B. J. West, Phys. Rev. E 70, 036105 (2004).

[43] L. de Arcangelis, Eur. Phys. J. Special Topics 205, 243 (2012).

[44] V. F. Pisarenko and D. Sornette, Eur. Phys. J. Special Topics 205, 95 (2012).

[45] The European Physical Journal-Special Topics (EPJ-Special Topics) has published a special issue of 21 papers devoted to the concept of Dragon King. We limit ourselves to quote only the papers of Refs. [43] and [44].

[46] G. Longo and M. Montévil, Front. Physiol. 3, 39 (2012).

[47] M. Turalska, E. Geneston, B. J. West, P. Allegrini, and P. Grigolini, Front. Physiol. 3, 52 (2012). 